\title{
Interface design enabled manufacture of giant metallic glasses
}

\author{
Hongzhen $\mathrm{Li}^{1 \dagger}$, Zhen $\mathrm{Li}^{1,2 \dagger}$, Jian Yang ${ }^{1}$, Hai Bo Ke ${ }^{3}$, Baoan $\mathrm{Sun}^{3,4}$, Chen Chen Yuan ${ }^{5}$, Jiang $\mathrm{Ma}^{1^{*}}$, \\ Jun Shen ${ }^{1}$ and Wei-Hua Wang
}

\begin{abstract}
Developing materials with excellent properties has been the untiring pursuit of mankind. Metallic glasses (MGs) would be the ideal metallic materials if their size could be scaled up to be comparable to traditional metals. To address this challenge, a variety of approaches have been attempted over the past decades, including thermodynamicsbased alloy, 3D printing and the recent artificial intelligenceguided optimal alloy. In this study, a facile and flexible route was demonstrated to manufacture giant MGs (GMGs) with diameters more than $100 \mathrm{~mm}$ through the thermo-joining process. The jointed GMG samples feature almost the same performance as the as-cast ones. The ability of manufacturing complex 3D components such as the Chinese Zodiacs was also demonstrated. Our approach might overcome the longstanding problem of glass forming ability (GFA) limitations in alloy systems and pave new concept and route to fabricate size unlimited MGs.
\end{abstract}

Keywords: giant metallic glass, glass forming ability, thermomanufacture, interface design

\section{INTRODUCTION}

Glasses and metals are two kinds of essential materials which play critical roles both in scientific research and daily life in the long history of mankind. As a combination, metallic glasses (MGs) possess the advantages of glasses and metals, which have gained a great deal of attention ever since being discovered [1-11]. The high specific strength, large elastic limit, excellent wear and corrosion resistance along with other remarkable en- gineering properties make these materials very promising in various engineering applications, such as sports goods, biomedical and electronic devices $[2,3,5,12]$. Nevertheless, different from the common glass forming materials, such as polymers, silicates, or molecular liquids, the crystallization rates of the known glass-forming metallic liquids remain orders of magnitude higher [13]. Consequently, glass forming ability (GFA) remains a long-standing issue for the fundamental research of $M G$ and a bottleneck for the potential applications of bulk MGs. In general, the GFA of metallic liquids is composition-sensitive and, in some cases, could be easily altered by the minor addition of a particular alloying element [14,15]. Many criteria have been proposed for the selection of alloy compositions that would favor glass formation [3]; however, universal criterion is still lacking. At present, superior GFA is only found in a limited number of MG systems, and the largest diameter cast into a fully glassy state appears to be $80 \mathrm{~mm}$ [16], which is noble metal-based, making this kind of material far from the requirement of engineering applications.

Various methods have been developed over the past decades to understand and improve the GFA of MGs or to overcome the GFA limit of existing MG formers. These include the approaches based on thermodynamics [3], the artificial intelligence-guided machine learning [17], the high throughput component selection [18], the 3D printing $[19,20]$, the ultrasonic welding [21-25], and the spark plasma sintering (SPS) method [26]. Among them, the thermoplastic joining seems to be a promising

\footnotetext{
${ }^{1}$ College of Mechatronics and Control Engineering, Shenzhen University, Shenzhen 518060, China

${ }^{2}$ College of Physics and Optoelectronic Engineering, Shenzhen University, Shenzhen 518060, China

${ }^{3}$ Songshan Lake Materials Laboratory, Dongguan 523808, China

${ }^{4}$ Institute of Physics, Chinese Academy of Sciences, Beijing 100190, China

${ }^{5}$ School of Materials Science and Engineering, Jiangsu Key Laboratory for Advanced Metallic Materials, Southeast University, Nanjing 211189, China

${ }^{\dagger}$ These authors contributed equally to this work.

* Corresponding author (email: majiang@szu.edu.cn)
} 
method to synthesize large-sized MGs considering that the processing temperature regime is much lower than the melting temperature $[27,28]$. However, the MGs fabricated by the joining method often suffer severe detrition on the properties due to the defects on the interfaces. The massive interfaces including the oxidation layer between the powders act as the key obstacles to the metallic bonding. Furthermore, the pore defects and crystallization cannot be prevented [29], even though high pressure and high temperature have been applied in the power-to-bulk strategy. In general, the GFA, in other words, the size of MG remains as the Achilles' heel of this kind of materials for their application as engineering materials.

In this study, we developed a facile and flexible interface-design route to conquer the oxidation layer during the joining of MGs in the thermal plastic forming regime. Based on this method, giant MG (GMG) with a diameter of more than $100 \mathrm{~mm}$ can be manufactured through the designed thermal process out of feedstocks with designed interlocking structures. The GMG has almost the same performance with the as-cast one. This strategy could overcome the GFA limit for MGs.

\section{EXPERIMENTAL SECTION}

\section{Preparation of MG plates}

A typical system of La-based MG with the element composition of $\mathrm{La}_{62} \mathrm{Al}_{14} \mathrm{Cu}_{24}$ was chosen for present research. The critical glass forming size was $5 \mathrm{~mm}$ in previous report [30]. According to the differential scanning calorimetry (DSC) curve of the as-cast shown in Fig. S1, the temperature of the supercooled liquid region of Labased MG is $400-445 \mathrm{~K}$. The MG plates were prepared by the conventional water-cooled copper mold casting process. To accomplish the specially designed experiments, the plates were cut into pieces with distinct lengths and shapes to form the interlocking structures.

\section{Thermo-manufacture process}

The thermo-manufacture process of GMG was conducted in a customized hot embossing machine equipped with precise temperature and motion control systems. The stacked MG plates were firstly placed into the furnace and covered tightly, the temperature was ramped into the supercooled liquid region of MG, and then, the force was applied on the viscous specimens to form them into big sizes or complex shapes. To avoid the risk of being crystallized, the customized machine had a rapid cooling system, which could lower the temperature of the samples in seconds. In the process of manufacturing GMG, the maximum temperature of the GMG sample measured by a thermocouple was $415 \mathrm{~K}$, and the generated pressure $(P)$ was $14.2 \mathrm{MPa}$.

\section{Characterization}

The amorphous nature of all the samples in present research was ascertained by the X-ray diffraction (XRD; Rigaku MiniFlex 600) with $\mathrm{Cu} \mathrm{Ka}$ radiation and DSC (Perkin-Elmer DSC-8000) at a heating rate of $20 \mathrm{~K} \mathrm{~min}^{-1}$. The mechanical performance was measured on the electromechanical SANS equipment at a constant strain rate of $10^{-4} \mathrm{~s}^{-1}$. The nano-scale mechanical performance was tested on a nanoindentation testing system (Hysitron TI950) with a Berkovich tip. The micro morphologies and elemental distributions of samples were characterized by FEI Quanta 450 FEG scanning electron microscope (SEM), and JEOL 2100F transmission electron microscope (TEM) equipped with energy disperse spectroscopy (EDS). The TEM sample was prepared by the FEI Scios focused ion beam/scanning electron microscope (FIB/ SEM) system. The microhardness of the as-cast and manufactured MG specimens was tested on the FMARS9000 automatic micro hardness instrument. Under the control of program, the diamond indenter was pressed into the surface of the specimen along its axial direction with a force of $0.49 \mathrm{~N}$, and then the force was held for $10 \mathrm{~s}$. After the size of the indentation on MG surface was automatically measured, the Vickers hardness could be calculated by the built-in application. The densities of the specimens were measured by the buoyancy method. The weight of specimen was measured in the air with an electronic balance (Sartorius Quintix35-1CN, measurement accuracy $0.01 \mathrm{mg}$ ), and was marked as W1. After that, the weight of sample in the analytical pure alcohol was measured and marked as W2. According to the formula $\rho=W_{1} \cdot \rho_{1} /\left(W_{1}-W_{2}\right)$, where the density of the analytical pure alcohol is $0.790 \mathrm{~g} \mathrm{~mL}^{-1}$, the density of the specimen could be calculated. To obtain the reliable results, the density measurements were repeated five times for each specimen. A Computed tomography (CT, Sanying precision instruments-nano Voxel 3000d) device was used to perform three-dimensional (3D) visual characterization of the welded sample. The characteristics and dimensions of the internal defects of the sample could be clearly and accurately displayed.

\section{Finite element (FE) simulation}

The FE simulations on the deformation and bonding of two MG samples in the supercooled liquid region were 
carried out by using Abaqus. The principles of plastic flow and Drucker-Prager evolution were adopted to establish 3D FE models for metal base and oxidation layer, respectively. The hardness (8-12 GPa) and Young's modulus (101-125 GPa) of the oxidation layers obtained from nano-indentation tests and the viscosity $\left(5 \times 10^{8} \mathrm{~Pa} \mathrm{~s}\right)$ of the supercooled La-MG liquid [31] were taken as input parameters of the models. To understand the basic bonding mechanisms, the contact interfaces of the samples were simplified as plastic metal surfaces partitioned by two oxidation layers with a thickness of $160 \mathrm{~nm}$, and the loading processes were performed on the initial samples with a clearance of $1 \mathrm{~mm}$ to the boundary constraints.

\section{RESULTS AND DISCUSSION}

\section{Interface design strategy for joining GMGs}

A strong physical bond of two surfaces requires atomic contact and surfaces free of contaminants, and therefore, metallic bond between the plate layers during the manufacturing process is required to form large-size MGs in the real sense. For MGs, oxidation layers act as a diffusion barrier and in general render metallic bonding difficult, since the manufacturing process was conducted in the air without any oxidation protection. To detect the motion and change of the oxidation layer, FE method was used to simulate the thermo-manufacture process, which is expected to confirm the experimental observations. To conduct the FE analyses, some parameters such as the thickness and mechanical values of the oxidation layer on MG surface were pre-measured by the EDS pattern and the nanoindentation, respectively. The thickness of the oxidation layer was about $80 \mathrm{~nm}$ (see Fig. S2). The nanoindentation experiments were conducted on the Labased MG samples to obtain the hardness and Young's modulus of the oxidation layers, and the hardness and Young's modulus values are in the range of 8-12 GPa and 101-125 GPa, respectively (see Figs S3 and S4). To eliminate the influence of the relatively soft metal base [32], only the indentation depth in $1 / 10-1 / 7$ of the oxidation layer thickness was adopted.

Generally, the thermo-manufacturing process of GMG can be reduced to the flow of viscous liquid coating with a thin rigid oxidation layer. Only the rigid layer was broken, while can the pristine liquid combine each other to form the metallic bond and obtain the intact and dense GMG? In the loading of interlocking structures designed in this study, the stress concentration appears in the center of interface due to the spatially nonuniform structures, as shown in left column of Fig. 1a, and thus the oxidation layer in that area will be firstly broken, and the metal base then can locally contact and bond with each other in the center of the structure. Meanwhile, the material volume and deformation in the center of interlocking structures are larger than that of the surrounding areas. Therefore, with the increase of loading pressure and deformation, the locally bonded metal will laterally flow from the center to the periphery of structures, which can remove the fractured oxidation layer from the bonding interface, and further extend the contact areas of the metal base, significantly improving the bonding quality of GMG samples. However, in the conventional bonding process, as shown in the right column of Fig. 1a, the loading stress tends to distribute uniformly on the interface of the samples, and thus the oxidation layer is difficult to break under the same pressure. In addition, this loading mode will not facilitate the lateral flow of metal base but induce multiple small fracture areas of oxidation layer, and thus the removal of fractured oxidation by unidirectional flow cannot be effectively achieved, leading to residual fractured oxidation on the interface, which will hinder the reliable bonding of the MG samples. The whole process can be illustrated in Fig. 1b. Firstly, the oxidation layer contacted and locally broke under pressure, and then the pristine viscous liquid met together and formed metallic bonding, resulting in a big one welded together.

\section{Thermo-joining of GMGs}

The schematic diagram of the thermo-manufacture process is illustrated in Fig. 2a. The multi-layer MG plates were stacked together by the designed interlocking architecture to facilitate the fully metallic bonding of interfaces between these plates. Fig. $2 \mathrm{~b}$ and its inset show the comparison of the thermo-manufactured GMG with a diameter of $20 \mathrm{~mm}$ and the as-cast sample with critical diameter of $5 \mathrm{~mm}$. To fabricate this GMG, six layers with interlocking structures were used. The amorphous nature of the manufactured sample was ascertained by the XRD and DSC, which were presented in Figs S5 and S1 in the supplementary materials. The diameter and height of the manufactured sample used in Fig. S5 is $8 \mathrm{~mm}$, and the weight of the manufactured sample used in the DSC test in Fig. S1 is $14.55 \mathrm{mg}$.

The high-resolution CT equipment with real spatial resolution of $0.5 \mu \mathrm{m}$ was applied to detect the defects on the holistic scale. Owing to the usage of high voltage Xray source with micro focal spot and highly sensitive detector, the CT can display the inside of the sample 

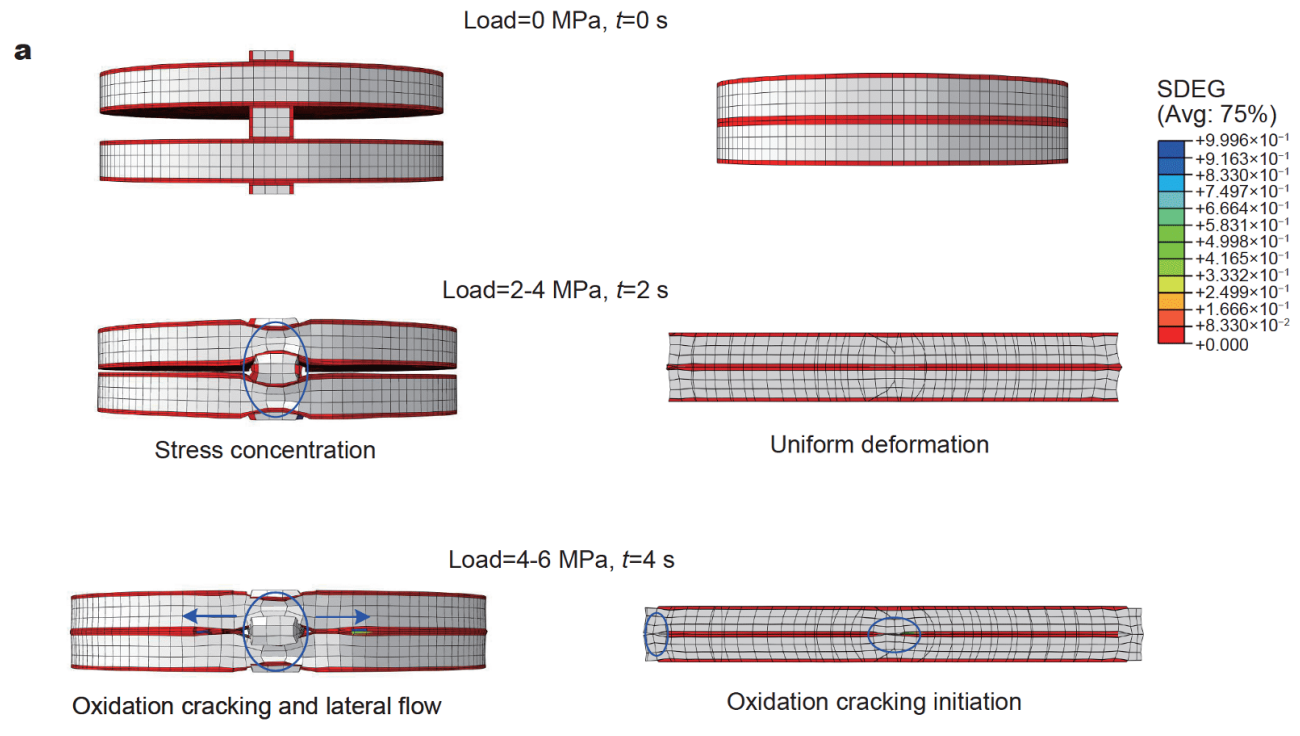

Oxidation cracking and lateral flow

Oxidation cracking initiation
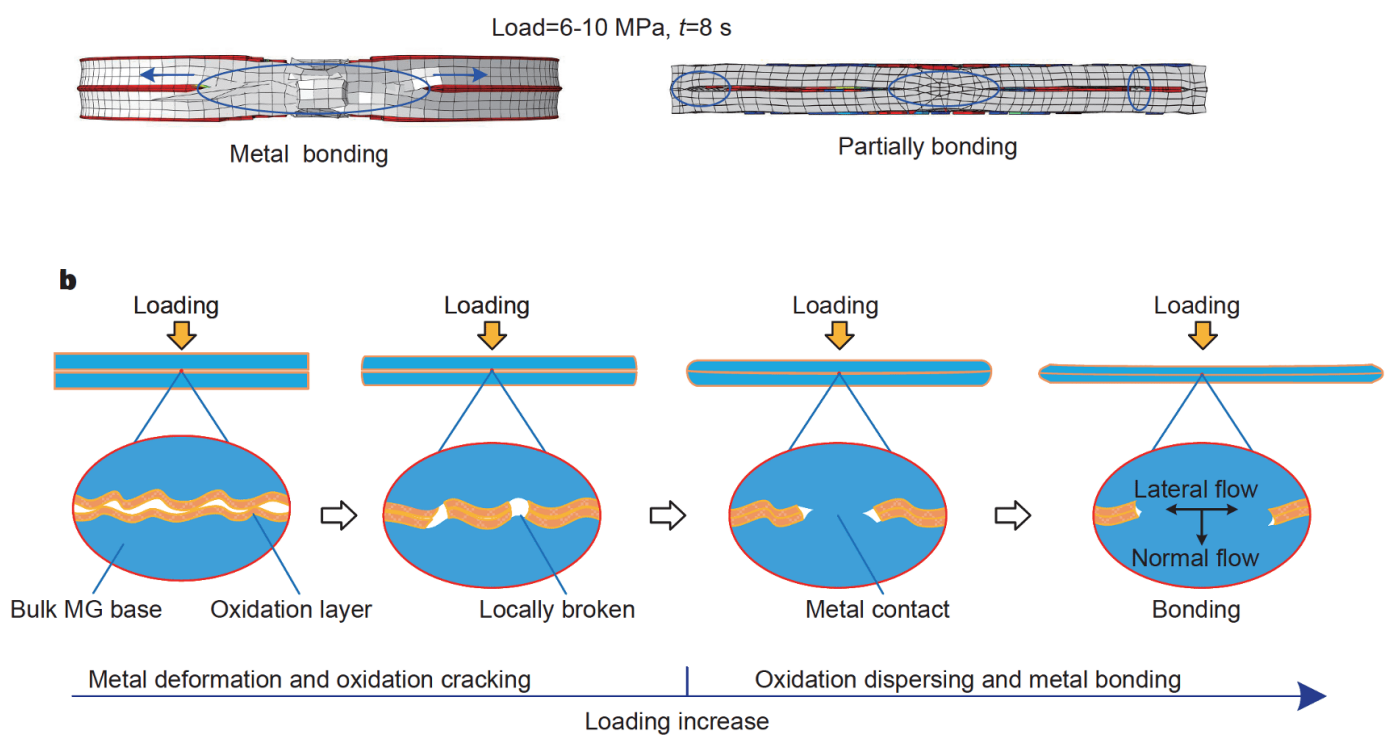

Figure 1 The crack of the oxidation layer and bonding of metal base. (a) The deformation of metal base and fracture of the oxidation layer of La-MG samples along with the increase of normal pressure loads with different initial structures. The left column shows the interlocking structures, and the right column shows the conventional layout. (b) Schematic of the mechanism of metallic bonding in the supercooled liquid region of MGs.

clearly, such as cracks, holes and defects. Limited by the penetration depth of the device, the thermo-manufactured sample with a diameter of $10 \mathrm{~mm}$ was chosen for the detection of CT and the cross-sectional CT images at different cutting positions from the outer to the center are presented in Fig. 2c. One can see that the sample is quite dense at the continuous cutting positions, and the results reflect the thermo-manufacture is a flexible method to synthetize GMGs with fully intact bonding.
To date, the maximum diameter of cast MGs reported was only $80 \mathrm{~mm}$ [33] and based on the noble metal palladium, greatly weakening their practical significance. On the other hand, most promising non-noble systems were restricted by the critical diameter less than $10 \mathrm{~mm}$ [34]. Fig. 3a summarizes the size development of MGs along with years since it was discovered in 1960s [35], experiencing film, ribbon to bulk states, with details list in Table S1. If we plot the maximum diameters at different years, 

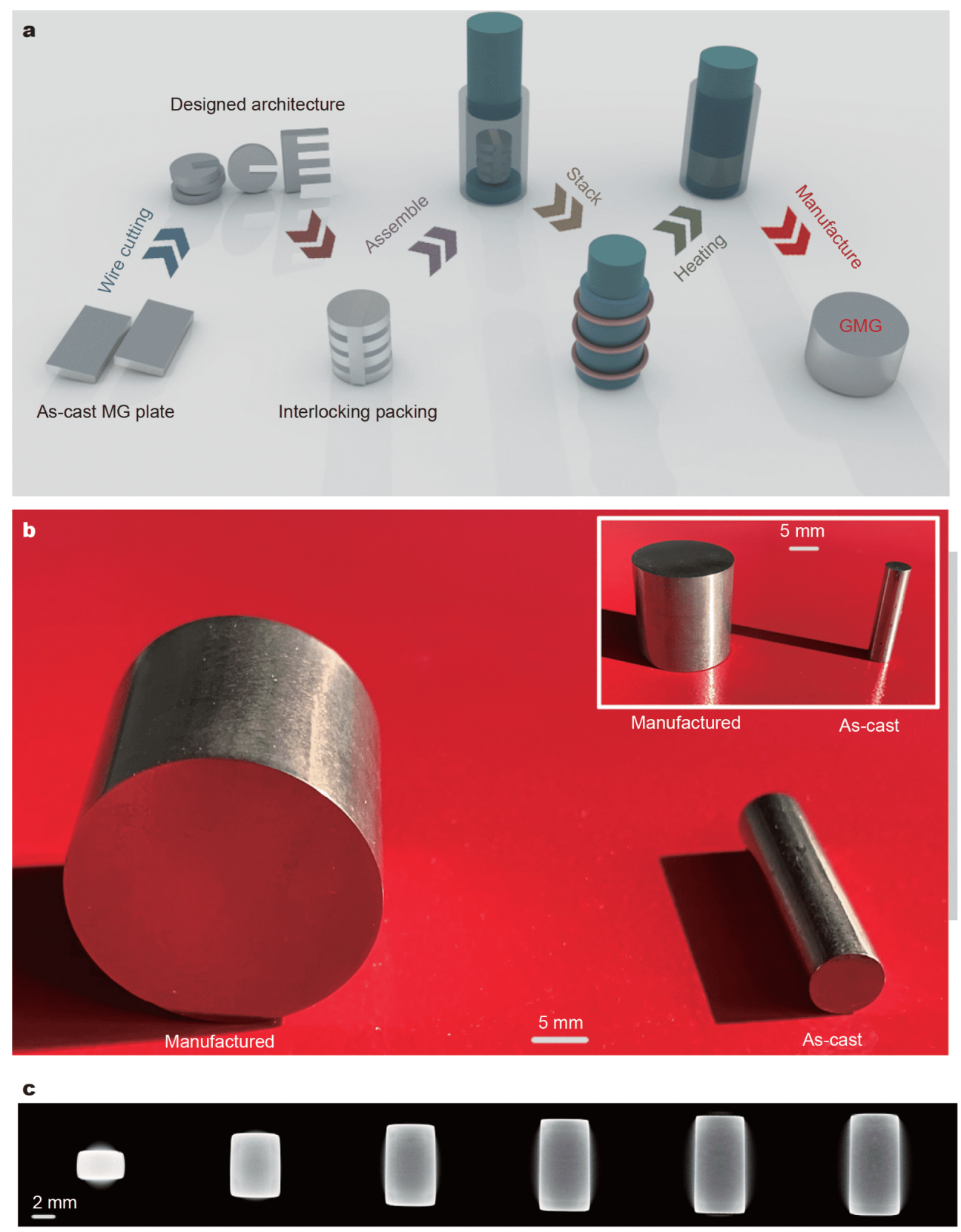

Figure 2 Manufacturing process of GMG. (a) The schematic diagram of the thermo-manufacture process. (b) The comparison of the thermosmanufactured big metallic glass with diameter and thickness of $20 \mathrm{~mm}$ and the as-cast sample with critical diameter of $5 \mathrm{~mm}$. The inset shows the different view angle. (c) Cross-sectional CT images at different cutting positions.

the trend of MG size can be included in the inset of Fig. 3a. The period when there was a rapid development of MG size is just in coincidence with the prosperity of this field. Obviously, to manufacture and design new generation of large-size MGs (diameter $>100 \mathrm{~mm}$ ) is of great significance both in scientific research and engineering applications.
Fig. 3b, c show the photograph of the as-cast and thermo-manufactured MGs with diameters of $20 \mathrm{~mm}$ and $100 \mathrm{~mm}$, respectively, where a sharp visual contrast can be observed. More than forty layers of MG plates with interlocking structures were used to manufacture fully amorphous GMG with diameter of more than $100 \mathrm{~mm}$ out of the system only with critical size of $5 \mathrm{~mm}$. Fully 

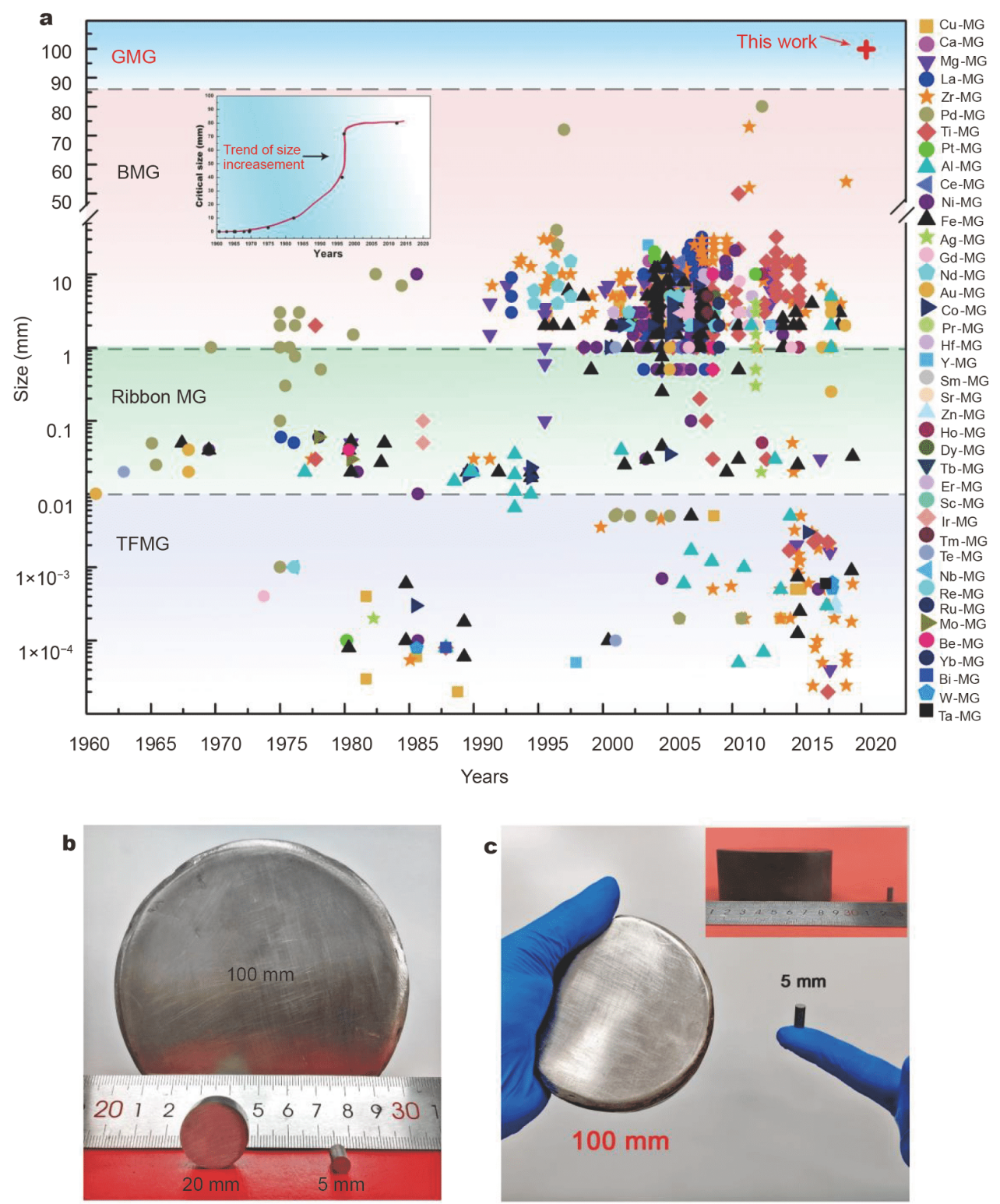

Figure 3 The size development of MG with years. (a) Since discovered in 1960s, MG has experienced film, ribbon to bulk states, ranging from nanometers to tens of millimeters. The inset shows the trend of maximum diameters of MGs at different years. Compared with the existing MGs, present work offers MGs larger than $100 \mathrm{~mm}$ in diameter. (b, c) The photographs of the manufactured large-size MG and its comparison with the ascast one.

amorphous and dense sample can be fabricated, which is more exceptional than other additive techniques. Our approach is a promising method to manufacture MGs with unlimited sizes, and could be a breakthrough to the glass forming limit of MGs.

\section{Structure characterization and mechanical performance}

The densities of the as-cast and thermo-manufactured samples were measured, with the values of $6.088 \pm 0.030$ and $6.082 \pm 0.055 \mathrm{~g} \mathrm{~cm}^{-3}$, respectively, as shown in Fig. $4 \mathrm{a}$. The diameter and height of the manufactured samples were both $8 \mathrm{~mm}$. Only $0.1 \%$ error of density is observed in the average level, implying they are as dense as each other. To further investigate the intrinsic structure of the fabricated MG, TEM was applied to observe the details near the bonding interface, which is essential for the 

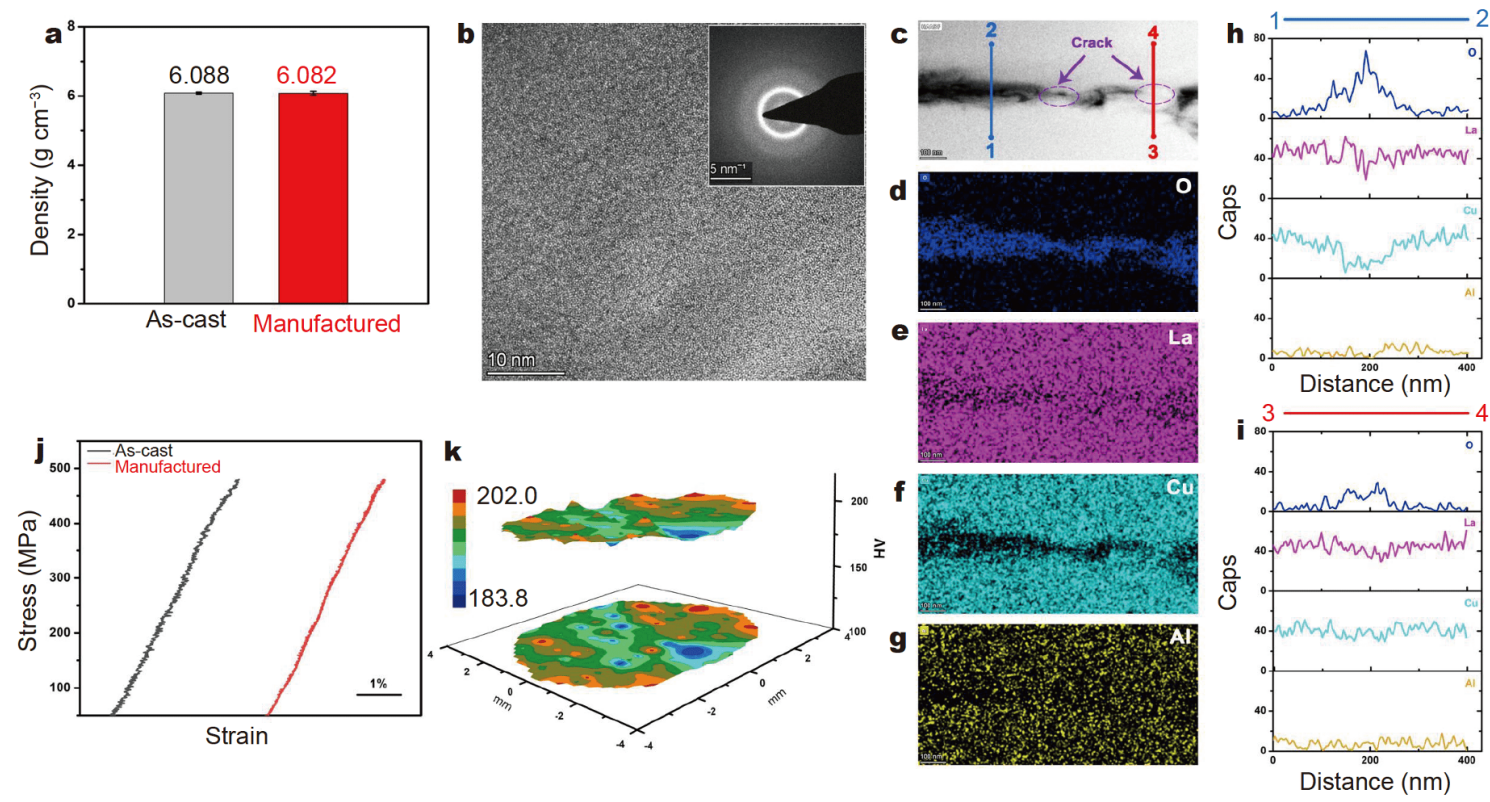

Figure 4 Characterizations of the manufactured GMG. (a) The densities of the as-cast and thermo-manufactured samples. (b) The high-resolution image and the diffraction pattern near the bonding interface region. (c) The crack of oxidation layer during the manufacture process. (d-g) The EDS of $\mathrm{O}, \mathrm{La}, \mathrm{Cu}$ and $\mathrm{Al}$. (h, i) The EDS line scanning of lines 1-2 and 3-4 in (c). (j) The compression tests of the as-cast and manufactured samples. (k) The $3 \mathrm{D}$ data plot and its projection of microhardness test of the manufactured sample.

manufacturing. Fig. $4 \mathrm{~b}$ presents the high-resolution image and the diffraction pattern, indicating the fully amorphous structure. To achieve metallic bonding and realize the manufacture of GMGs, it should overcome the barrier of thin oxidation layers between the stock plates during the thermal process. Otherwise, the strong bonding cannot be obtained in the intact samples. Fig. 4c shows the crack of oxidation layer during the manufacture process. Obviously, the rigid but fragile oxidation cracks into sections when subjected to a certain force. The elemental analysis is also presented in Fig. 4d-g, clearly revealing the metallic bonding and oxidation crack during the manufacturing process. Fig. $4 \mathrm{~h}$, i give the line scanning of lines $1-2$ and $3-4$ in Fig. $4 c$, and it can be seen that metallic elements were separated by oxygen in line 1-2; however, there is a uniform distribution at the crack region in line 3-4, which is the solid evidence showing the real bonding of different bulk MG plates with interlocking structures.

Mechanical performance is a key issue for the manufactured GMG. To verify the influence that the manufacture process could bring on the mechanical property, the compression tests of the as-cast and manufactured samples were performed, as shown in Fig. 4j. The as-cast and manufactured compression test samples are cylinder with a diameter of $2 \mathrm{~mm}$ and a height of $4 \mathrm{~mm}$. For the convenience of compression test, the sample of the manufactured one was cut by a precise wire cutting machine from the GMG. Both of the samples reveal typical brittle fracture with exactly similar strength of about $480 \mathrm{MPa}$, and no degradation of macroscopic performance was found. Microhardness tests were also conducted to reveal the surface mechanical condition of the manufactured sample. More than 200 points were collected in an area of diameter $8 \mathrm{~mm}$ crossing the boundary between plate layers. The 3D data plot and its projection were presented in Fig. 4k. Only small fluctuation can be found in the microhardness values, indicating the uniform surface property and no bonding gap.

\section{D components fabrication}

Furthermore, components with complex 3D shapes can be shaped using the thermo-manufacturing process. Owing to the brittle nature, MGs are not easy to be machined into specific required shapes, though they have superior properties. We show here that various components can be manufactured and formed simultaneously through our method, and this process is an integration of manufacturing and forming process and can also efficiently fabricate complex structures. We designed the traditional Chinese Zodiac of twelve different animals (See the design drawing in Fig. S6), as presented in 


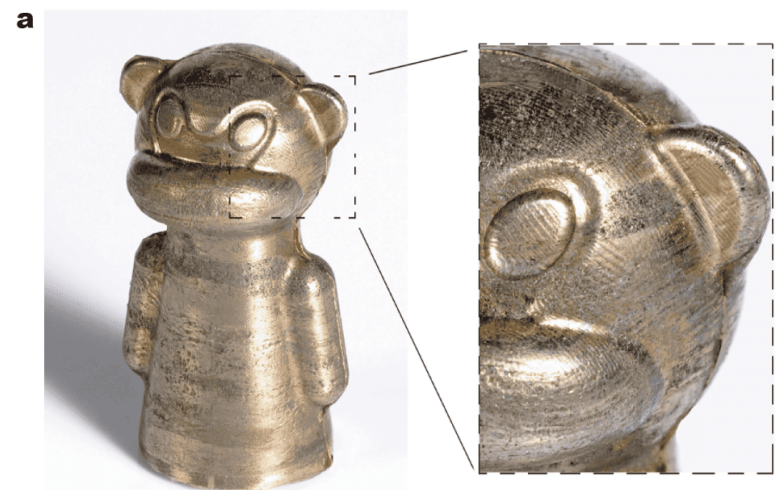

b

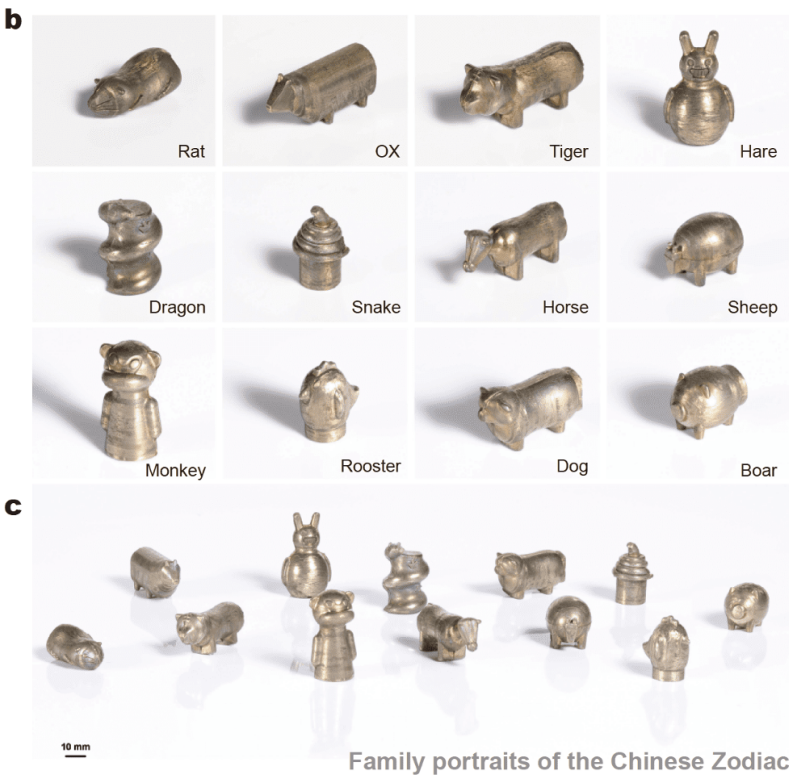

Figure 5 The integration of manufacturing and forming. The photographs of traditional Chinese Zodiac of twelve different animals, in solo and family portraits styles, shown in $(\mathrm{a}-\mathrm{c})$, respectively.

Fig. 5a-c. Fig. 5a exhibits the Monkey character and its details. The vivid profile demonstrates the forming accuracy. Fig. 5b, c show the complete set of the Zodiacs, in solo and family portraits styles. Multifarious complex structures of these animals with larger size than the critical dimension, including curved profile, sharp angles, such as the beard of rat, the horns of OX, the ears of hare, the eyes of the monkey and so on, were precisely manufactured. These results indicate that the thermo-manufacture can scale up the size and construct the shape simultaneously.

\section{CONCLUSION}

In summary, we have successfully manufactured GMGs with diameter larger than $100 \mathrm{~mm}$ through interface design strategy without degradation of mechanical performance. The complex 3D components can be produced by the proposed integrated manufacture and forming process. This method can overcome the GFA limitations of alloys to fabricate large-size MGs, which opens up a new route towards the engineering applications of these advanced materials.

Received 22 September 2020; accepted 11 November 2020; published online 4 January 2021

1 Johnson WL. Bulk glass-forming metallic alloys: Science and technology. MRS Bulletin, 1999, 24: 42-56

2 Wang WH, Dong C, Shek CH. Bulk metallic glasses. Mater Sci Eng-R-Rep, 2004, 44: 45-89

3 Inoue A. Stabilization of metallic supercooled liquid and bulk amorphous alloys. Acta Mater, 2000, 48: 279-306

4 Demetriou MD, Launey ME, Garrett G, et al. A damage-tolerant glass. Nat Mater, 2011, 10: 123-128

5 Telford M. The case for bulk metallic glass. Mater Today, 2004, 7: 36-43

6 Guan P, Chen M, Egami T. Stress-temperature scaling for steadystate flow in metallic glasses. Phys Rev Lett, 2010, 104: 205701

7 Nieh T. Plasticity and structural instability in a bulk metallic glass deformed in the supercooled liquid region. Acta Mater, 2001, 49: 2887-2896

8 Schuh CA, Hufnagel TC, Ramamurty U. Mechanical behavior of amorphous alloys. Acta Mater, 2007, 55: 4067-4109

9 Sun BA, Yu HB, Jiao W, et al. Plasticity of ductile metallic glasses: A self-organized critical state. Phys Rev Lett, 2010, 105: 035501

10 Greer AL. Metallic glasses...on the threshold. Mater Today, 2009, 12: $14-22$

11 Ma J, Zhang XY, Wang DP, et al. Superhydrophobic metallic glass surface with superior mechanical stability and corrosion resistance. Appl Phys Lett, 2014, 104: 173701

12 Schroers J. Processing of bulk metallic glass. Adv Mater, 2010, 22: 1566-1597

13 Johnson WL, Kaltenboeck G, Demetriou MD, et al. Beating crystallization in glass-forming metals by millisecond heating and processing. Science, 2011, 332: 828-833

14 Wang W. Roles of minor additions in formation and properties of bulk metallic glasses. Prog Mater Sci, 2007, 52: 540-596

15 Lu ZP, Liu CT. A new glass-forming ability criterion for bulk metallic glasses. Acta Mater, 2002, 50: 3501-3512

16 Inoue $\mathrm{A}$, Nishiyama $\mathrm{N}$, Kimura $\mathrm{H}$. Preparation and thermal stability of bulk amorphous $\mathrm{Pd}_{40} \mathrm{Cu}_{30} \mathrm{Ni}_{10} \mathrm{P}_{20}$ alloy cylinder of $72 \mathrm{~mm}$ in diameter. Mater Trans JIM, 1997, 38: 179-183

17 Sun YT, Bai HY, Li MZ, et al. Machine learning approach for prediction and understanding of glass-forming ability. J Phys Chem Lett, 2017, 8: 3434-3439

18 Li MX, Zhao SF, Lu Z, et al. High-temperature bulk metallic glasses developed by combinatorial methods. Nature, 2019, 569: 99-103

19 Pauly S, Löber L, Petters R, et al. Processing metallic glasses by selective laser melting. Mater Today, 2013, 16: 37-41

20 Gibson MA, Mykulowycz NM, Shim J, et al. 3D printing metals like thermoplastics: Fused filament fabrication of metallic glasses. 
Mater Today, 2018, 21: 697-702

21 Ma J, Yang C, Liu X, et al. Fast surface dynamics enabled cold joining of metallic glasses. Sci Adv, 2019, 5: eaax7256

22 Liang X, Zhu XL, Li X, et al. High-entropy alloy and amorphous alloy composites fabricated by ultrasonic vibrations. Sci ChinaPhys Mech Astron, 2020, 63: 116111

23 Li Z, Huang Z, Sun F, et al. Forming of metallic glasses: mechanisms and processes. Mater Today Adv, 2020, 7: 100077

24 Li X, Liang X, Zhang Z, et al. Cold joining to fabricate large size metallic glasses by the ultrasonic vibrations. Scripta Mater, 2020, 185: $100-104$

25 Sohrabi S, Li MX, Bai HY, et al. Energy storage oscillation of metallic glass induced by high-intensity elastic stimulation. Appl Phys Lett, 2020, 116: 081901

26 Li XP, Yan M, Imai H, et al. Fabrication of $10 \mathrm{~mm}$ diameter fully dense $\mathrm{Al}_{86} \mathrm{Ni}_{6} \mathrm{Y}_{4.5} \mathrm{Co}_{2} \mathrm{La}_{1.5}$ bulk metallic glass with high fracturestrength. Mater Sci Eng-A, 2013, 568: 155-159

27 Chen W, Liu Z, Schroers J. Joining of bulk metallic glasses in air. Acta Mater, 2014, 62: 49-57

28 Ojeda Mota RM, Liu N, Kube SA, et al. Overcoming geometric limitations in metallic glasses through stretch blow molding. Appl Mater Today, 2020, 19: 100567

29 Xie G, Zhang W, Louzguine-Luzgin DV, et al. Fabrication of porous $\mathrm{Zr}-\mathrm{Cu}-\mathrm{Al}-\mathrm{Ni}$ bulk metallic glass by spark plasma sintering process. Scripta Mater, 2006, 55: 687-690

30 Jiang QK, Zhang GQ, Chen LY, et al. Glass formability, thermal stability and mechanical properties of La-based bulk metallic glasses. J Alloys Compd, 2006, 424: 183-186

31 Okumura $\mathrm{H}$, Inoue $\mathrm{A}$, Masumoto T. Glass transition and viscoelastic behaviors of $\mathrm{La}_{55} \mathrm{Al}_{25} \mathrm{Ni}_{20}$ and $\mathrm{La}_{55} \mathrm{Al}_{25} \mathrm{Cu}_{20}$ amorphous alloys. Mater Trans JIM, 1991, 32: 593-598

32 Jönsson B, Hogmark S. Hardness measurements of thin films. Thin Solid Films, 1984, 114: 257-269

33 Nishiyama N, Takenaka K, Miura H, et al. The world's biggest glassy alloy ever made. Intermetallics, 2012, 30: 19-24

34 Long Z, Wei H, Ding Y, et al. A new criterion for predicting the glass-forming ability of bulk metallic glasses. J Alloys Compd, 2009, 475: 207-219

35 Klement Jun. W, Willens RH, Duwez P. Non-crystalline structure in solidified gold-silicon alloys. Nature, 1960, 187: 869-870

Acknowledgements The work was supported by the Key Basic and Applied Research Program of Guangdong Province, China (2019B030302010), the National Natural Science Foundation of China (51871157), the Science and Technology Innovation Commission of Shenzhen (JCYJ20170412111216258), the National Key Research and Development Program of China (2018YFA0703605). The authors are grateful for the helpful discussion with Dr. Wang C and also thank the assistance on microscope observation received from the Electron Microscope Center of Shenzhen University.

Author contributions Ma J, Shen J and Wang WH designed and supervised the work. Li H and Li Z conducted the experiments, carried out the transmission electron microscopy observation, and performed the preparation of metallic glass samples, calorimetry, Vickers microhardness, and X-ray diffraction tests as well as the modeling and simulations. Ma J, Li Z, Ke HB, Sun B, Yuan CC and Li H wrote and polished the manuscript. All authors contributed to the analyses and interpretation of the data, and the general discussion.
Conflict of interest The authors declare no competing financial interest.

Supplementary information Supporting data are available in the online version.

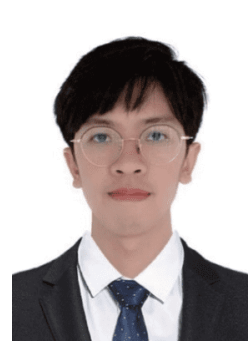

Hongzhen Li received his master degree in mechanical engineering from Shenzhen University (SZU) in 2020. Currently, he is a full-time researcher in the School of Mechatronics and Control Engineering, Shenzhen University. His research includes metallic glass, high-entropy alloys, advanced manufacturing and $3 \mathrm{D}$-printing.

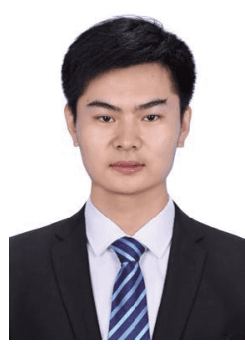

Zhen Li received his BSc degree in mechanical engineering from Henan Polytechnic University (HPU) in 2013, and PhD degree from Beihang University, in 2019. His research includes metallic glasses, high-entropy alloys, composite material, advanced manufacturing and surface engineering.

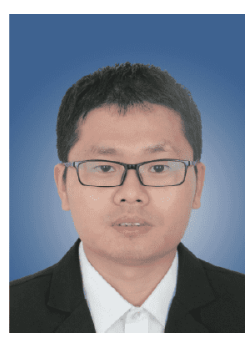

Jiang Ma received his BSc degree in materials science and engineering from Southeast University in 2009 and $\mathrm{PhD}$ degree from the Institute of Physics, Chinese Academy of Sciences (CAS), in 2014. He is currently a professor in the College of Mechatronics and Control Engineering, Shenzhen University. His research includes metallic glass, high-entropy alloy, micro/nano precision forming, functional surface fabrication and application.

\section{利用界面设计制造巨型金属玻璃}

李泓瑧 ${ }^{1 \dagger}$, 李真 ${ }^{1,2 \dagger}$, 杨剑 ${ }^{1}$, 柯海波 ${ }^{3}$, 孙保安 ${ }^{3,4}$, 袁晨晨 ${ }^{5}$, 马 将 ${ }^{1^{*}}$, 沈军 $^{1}$, 汪卫华 ${ }^{3,4}$

摘要 开发具有优良性能的材料一直是人类不解的追求. 如果将尺 寸放大到与传统金属相当的水平, 金属玻璃将是一种理想的金属 材料. 为了应对这一挑战, 在过去的几十年中, 研究学者们已经尝 试了多种方法，包括基于热力学的合金开发、3D打印以及基于人 工智能学习的合金优化设计新理念. 本文提出了一种简便、灵活 的界面设计理念来制造直径大于 $100 \mathrm{~mm}$ 的巨型金属玻璃(GMG), 通过该方法制造的巨型金属玻璃性能几乎与铸态样品相同. 此外, 利用该方法可制造复杂三维结构. 本文提出的方法为克服合金系 统中长期存在的玻璃形成能力(GFA)限制的问题, 制造大尺寸、复 杂结构金属玻璃开辟了新的思路和途径. 